\section{Darstellung von Aluminiumnitrid durch Umsetzung von Aluminium in einem nicht- isothermen Stickstoff/Chlor-Niederdruckplasma}

\author{
S. Veprek, C. BRENDEL und H. SchäFer
}

Anorganisch-Chemisches Institut

der Westfälischen Wilhelms-Universität Münster

(Z. Naturforsch. 24 a, 2025-2026 [1969] ; eingeg. am 24. Oktober 1969)

Neben anderen Methoden zur Darstellung von AlN 1, 2 kennt man die Synthese von AlN aus $\mathrm{AlCl}_{3}$ und $\mathrm{N}_{2}$ in einem Entladungsplasma. Diese kann in einem Hochdruckbogenentladungsplasma (z. B. ${ }^{3}$ ) oder in einem nicht-isothermen Niederdruckplasma ${ }^{4}$ ausgeführt werden. Wir haben die Chlorierung von $\mathrm{Al}$ und die Bildung von festem AlN im gleichen Reaktionsraum durchgeführt. Durch diesen strömte eine $\mathrm{Cl}_{2} / \mathrm{N}_{2}$-Mischung bei einem Gesamtdruck von 0,5 bis 5 Torr.

Im vorliegenden nicht isothermen Plasma befinden sich die Gasteilchen (Moleküle, Atome, Ionen, Elektronen) nicht im thermodynamischen Gleichgewicht. Wir nutzen die apparativen variierbaren Bereiche mit unterschiedlichen Plasmaparametern aus. Im Bereich A der Entladung wird bevorzugt $\mathrm{Cl}_{2}$ aktiviert $\left(\mathrm{Cl}_{n}{ }^{*}\right.$, d. h. $\mathrm{Cl}_{2}{ }^{*}$, $\left.\mathrm{Cl}, \mathrm{Cl}^{*} \ldots\right)$, was die Chlorierung des dort befindlichen Aluminiumblechs erleichtert. Bei geeigneter Versuchsführung kann außerhalb der Entladungszone $\mathrm{AlCl}_{3}$ abgeschieden werden. In diesem Entladungsbereich entsteht noch kein AlN.

Erst im anschließenden Teil B der Entladung wird $\mathrm{N}_{2}$ so stark aktiviert $\left(N_{z}{ }^{*}\right.$, Abkürzung für $\mathrm{N}_{2}{ }^{*}, \mathrm{~N}$, $\mathrm{N}^{*} \ldots$..), daß AlN gebildet wird. Qualitativ läßt sich das Geschehen durch folgende Modellgleichungen beschreiben:

$$
\begin{gathered}
\mathrm{Al}(\mathrm{f})+\frac{x}{n} \mathrm{Cl}_{n}{ }^{*}(\mathrm{~g})=\mathrm{AlCl}_{x}(\mathrm{~g}) \\
\operatorname{AlCl}_{x}(\mathrm{~g})+\frac{1}{z n} \mathrm{~N}_{z}^{*}(\mathrm{~g})=\mathrm{AlN}(\mathrm{f})+\frac{x}{n} \mathrm{Cl}_{n}{ }^{*} .
\end{gathered}
$$

Im Bereich A liegt die Reaktion (2) auf der linken, und im Bereich $B$ auf der rechten Seite. Es hängt vom Verhältnis $\mathrm{Cl}_{n}{ }^{*} / \mathrm{N}_{z}{ }^{*} \mathrm{ab}$, ob $\mathrm{AlCl}_{n}(\mathrm{~g})$ „stabil“ ist oder AlN abgeschieden wird.

Eine im Bereich der Entladung örtlich unterschiedliche Richtung der Reaktion (2), - an der Gaseintrittsstelle Verlauf nach links und später nach rechts muß zum chemischen Transport von AlN führen. Entsprechende Experimente mit AlN als Startmaterial stehen jedoch noch aus.

Im Plasma sind verschiedene Atomkombinationen von $\mathrm{Al}, \mathrm{N}$ und $\mathrm{Cl}$ möglich, jedoch ist $\mathrm{AlN}$ die einzige

Sonderdruckanforderungen an Prof. Dr. H. SchäFER, Anorgan.-Chemisches Institut der Westf. Wilhelms-Universität, D-4400 Münster, Gievenbecker Weg 9.

1 R. K. Willardson u. H. L. Goering, Compound Semiconductors, Vol. I, Reinhold Publ. Corp., New York 1962, Kap. 19. bei der hohen Temperatur der Unterlage (etwa $1100{ }^{\circ} \mathrm{C}$ ) abscheidbare (thermisch stabile) Verbindung. Eine weitere Selektion ergibt sich beim Aufbau des AlN-Gitters. Es besteht eine erhebliche Beweglichkeit (Oberflächendiffusion) von $\mathrm{Al}$ und $\mathrm{N}$, was zu einer gut geordneten AlN-Phase führt (Röntgen-Aufnahme). Dies verhindert (oder erschwert) die Aufnahme von Verunreinigungen in den Festkörper: Dient eine $\mathrm{Zn}, \mathrm{Fe}, \mathrm{Si}$, $\mathrm{Pb}, \mathrm{Mg}, \mathrm{Cu}$ enthaltende Al-Legierung als Startmaterial, so erhält man dennoch praktisch reines AlN. Spektralanalytisch sind nur Spuren von $\mathrm{Mg}$ und Si erkennbar; möglicherweise handelt es sich dabei um Substitution von $2 \mathrm{Al}$ durch $1 \mathrm{Mg}+1 \mathrm{Si}$.

\section{Experimente und Ergebnisse}

Die auf Abb. 1 dargestellte Anordnung diente zur Herstellung von pulverförmigem AlN oder von AlNKristallen. Abbildung 2 zeigt eine zur Abscheidung von AlN-Schichten auf einem Träger geeignete Anordnung.

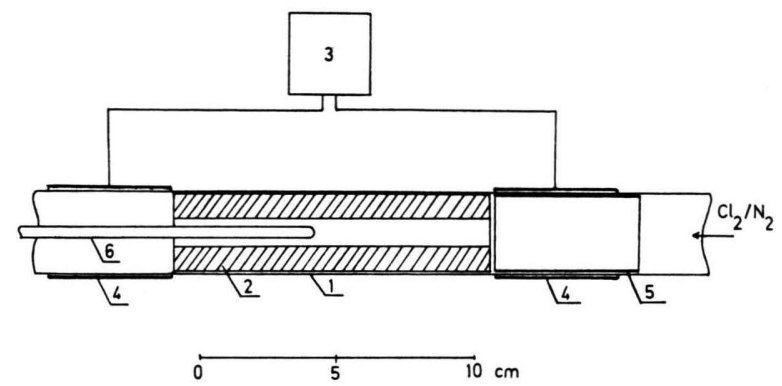

Abb. 1. Apparatur zur Darstellung von AlN-Pulver und -Kristallen.

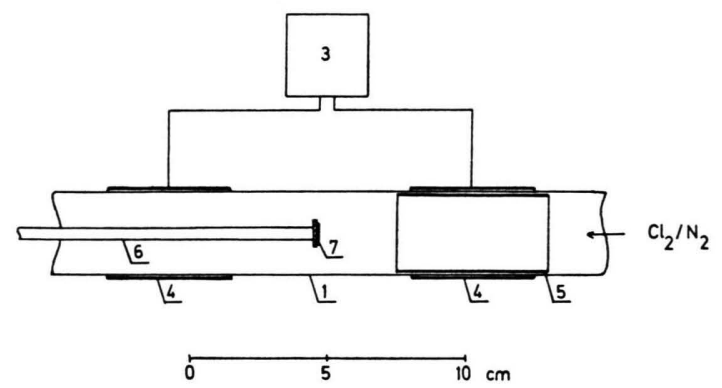

Abb. 2. Apparatur zur Darstellung von AlN-Schichten.

Folgende Angaben gelten für beide Anordnungen: Die Reaktion erfolgt in einem Quarzrohr (1), das von außen weder geheizt noch gekühlt wird. Als Aluminiumquelle dient das Aluminiumblech (5) mit der Dicke

2 A. A. Peletjuskin u. N. G. Slavina, Izv. Akad. Nauk SSSR, Ser. Neorg. Materialy 4, 893 [1968]. - G. A. Cox, D. O. Cummins, K. Kawabe u. R. H. Tredgold, J. Phys. Chem. Solids 28, 54 [1967].

3 Y. Haxashi, K. Okada u. S. Koide, Yogyo kyokai Shi 76, 307 [1968].

4 J. Pastrńák u. L. Součková, Phys. Status Solidi 3, K 71 [1963]. 
$1 \mathrm{~mm}$. Reinster Stickstoff wurde bei $-78,5{ }^{\circ} \mathrm{C}$ mit $\mathrm{Cl}_{2}$ gesättigt $(\Sigma P=1 \mathrm{~atm})$; danach betrug das Molverhältnis $\mathrm{N}_{2}: \mathrm{Cl}_{2} \cong 12: 1$. Dieses Gas strömte mit einer Geschwindigkeit von etwa $10^{2}$ bis $10^{3} \mathrm{ml}$ Torr $/ \mathrm{sec}$ in das Reaktionsrohr ein. Das am Ende des Reaktionsrohres angeschlossene Pumpsystem hielt im Rohr je nach Versuchsführung einen Druck von etwa 0,5 bis 5 Torr aufrecht.

Die Hochfrequenzentladung findet in dem Raum zwischen den äußeren Elektroden (4) statt. Der Hochfrequenzgenerator lieferte eine Frequenz von $80 \mathrm{MHz}$ mit einer Leistung bis zu 1,2 kW. Der Plasmazustand - insbesondere das wichtige Verhältnis $\mathrm{Cl}_{n}{ }^{*} / N_{z}{ }^{*}-$ läßt sich durch Hochfrequenzleistung, Gasdruck und Geometrie der Anordnung beeinflussen. Die Temperatur des neutralen Gases hängt außer von diesen Größen auch von der Wärmeisolierung ab.

Abscheidung von AlN-Pulver und -Kristallen (Abb. 1) : In das Quarzrohr wurden mehrere ineinander passende Korundrohre (2) (Degussit $\mathrm{Al}$ 23) gelegt, die den Innendurchmesser auf $10 \mathrm{~mm}$ verengten. Hierdurch wird die Hochfrequenzstromdichte erhöht und zugleich die Wärmeisolierung verbessert.

Pulverförmiges AlN scheidet sich an der Innenwand des Korundrohres ab. Dessen mit dem optischen Pyrometer gemessene Temperatur betrug hierbei etwa $1100{ }^{\circ} \mathrm{C}$. AlN-Kristalle (Größe bis zu $2 \mathrm{~mm}$ ) können auf einem einseitig geschlossenen Korundrohr (6) (Außendurchmesser $3-4 \mathrm{~mm}$ ), das in die Mitte der Entladung eingeführt wird, abgeschieden werden.

Das Korundrohr (6) konnte bei entsprechender Hochfrequenzleistung bis auf etwa $1600^{\circ} \mathrm{C}$ aufgeheizt werden. Jedoch waren so hohe Temperaturen bisher nicht ausnutzbar, weil oberhalb $1100{ }^{\circ} \mathrm{C} \mathrm{AlN}$ mit der $\mathrm{Al}_{2} \mathrm{O}_{3}$. Unterlage reagiert. Es ist dies natürlich nur eine Materialfrage.

AlN-Schichten wurden in der auf Abb. 2 dargestellten Anordnung erhalten. Hier bestand eine starke Radialabhängigkeit der Plasmaparameter. AlN schied sich auf der Unterlage (7) ab, die aus Quarz oder $\alpha-\mathrm{Al}_{2} \mathrm{O}_{3}$ bestand.

Alle gewonnenen AlN-Präparate geben gute RöntgenDiagramme vom Wurtzit-Typ. Aus Guinier-Aufnahmen ergab sich $a=3,11 \AA, c=4,98 \AA$ (Literaturwerte $a=$ $3,111 \AA, c=4,978 \AA^{5}$ ). Die Präparate sind glasklar und zeigen eine schwache, langsam abklingende blaue Lumineszenz.

Das Verfahren ist auf die Darstellung weiterer Nitride übertragbar. Hierbei sind zugleich weitere Aufschlüsse über das Reaktionsgeschehen zu erwarten.

5 G. A. Jeffrey, G. S. Parry u. R. L. Mozzi, J. Chem. Phys. 25, 1024 [1956]. 